\title{
MS37-04 | Determination of Elusive Crystal Structure of Solvate-Hydrate of Catechin by Crystal Structure Prediction and NMR Crystallography
}

Dudek, Marta (Centre of Molecular and Macromolecular Studies, Polish Academy of Sciences, Lodz, POL); Paluch, Piotr (Center of Molecular and Macromolecular Studies, Polish Academy of Sciences, Lodz, POL); Sniechowska, Justyna (Center of Molecular and Macromolecular Studies, Polish Academy of Sciences, Lodz, POL); Nartowski, Karol (Wroclaw Medical University, Wroclaw, POL); Trebosc, Julien (CNRS, Universite Lille-Nord-de-France, Lille, FRA); Potrzebowski, Marek (Center of Molecular and Macromolecular Studies, Polish Academy of Sciences, Lodz, POL); Day, Graeme (Univeristy of Southampton, Southampton, GBR)

The idea standing behind crystal structure elucidation using NMR crystallography is based on the assumption, that a significant difference in the agreement between experimental NMR parameters and theoretical ones (obtained for the proposed structural models) for the correct and incorrect crystal structures will be observed. While in many cases this assumption is true, with a considerable number of examples found in the literature, for multicomponent systems, such as solvates or hydrates, a different trend may be observed. In this work we present the limitations and applicability of a joint crystal structure prediction - NMR crystallography (CSP-NMR) approach to elucidate crystal structures of polyphenols epicatechin, catechin and procyanidin A-2, including a new, yet undescribed form, methanol solvate-hydrate of catechin. We demonstrate that when dealing with multicomponent systems, the observed difference in terms of RMS values for predicted ${ }^{1} \mathrm{H}$ solid-state NMR chemical shifts may not be as distinctive as this is the case of simpler crystal structures. Consequently, we try to answer the question how good agreement is good enough, when can we expect false-positive results to appear and can we learn from them about the molecule's crystallization preferences. Finally, we apply CSP-NMR protocol to determine crystal structure of a methanol hemisolvate - monohydrate of catechin, demonstrating a useful shortcut for the cases of crystal structure elucidation of multi-component flexible systems. 